\title{
Sequential QoS Mapping Model for Multi-layer Video Delivery in 802.11e Wireless Networks
}

\author{
Lamia Romdhani and Amr Mohamed \\ Computer Science and Engineering Department, Qatar University, Doha, Qatar \\ \{lamia.romdhani,amrm\}@qu.edu.qa
}

\begin{abstract}
Multi-layer video transmission over wireless networks poses numerous challenges due to symantec nature of the encoded video and the intrinsic dependency between the video layers, which, if not considered, may cause severe quality degradation. In this paper, we present a new analytical model leveraging cross layer design for distributed video layers mapping that optimizes the QoS of wireless video transmission over IEEE 802.11e EDCA (Enhanced Distributed Channel Access) priority queues. MRC (Multi-Resolution Coding) Layered video, divides video into a base layer and multiple enhancement layers. We aim to improve the perceived video quality, impacted by high channel contention, through mapping individual video layers into EDCA access categories in order to maximize the average number of reconstructed video layers. The proposed technique is based on a dynamic program that takes into account the EDCA parameters and the dependency nature of layered video delivery, leveraging an application-based metric that measures the symantec video quality. Our proposed technique makes use of a channel delay statistical model to estimate the expected useful video layers delivered. The performance of our optimized mapping technique is verified through extensive simulations. The obtained results illustrate significant trade-off between complexity and delivered video quality for canonical mapping schemes.
\end{abstract}

Index Terms-EDCA, Layered video, mapping strategy, analytical model.

\section{INTRODUCTION}

High video delivery quality over wireless networks is becoming a basic requirement of network architectures. Layered video, scalable video, and MRC, all refer to encoding techniques that fragment a video stream into a base layer and enhancement layers [1]. The base layer is necessary for decoding the video stream, whereas the enhancement layers improve its quality. Considering the one-hop broadcast nature of the wireless channel, all layers for all video users share and contend on the wireless medium. Thus, the enhancement layers reduce the bandwidth available to the base layer and further reduce the performance of poor receivers. The Quality-of-Service (QoS) of 802.11e [2] is achieved by providing different classes of frames with different priorities when accessing the radio channel. In the basic EDCA scheme, the video traffic is mapped automatically into two access classes. In this paper, we describe a distributed and adaptive cross-layer dynamic mapping techniques that map the arriving video packets into different EDCA Access Categories (ACs) to optimize layered video delivery by maximizing the expectation of the number of video layers.

Enhancing video delivery in different wireless network settings has been investigated extensively in the literature. Many techniques have been utilized to achieve such goal, including rate allocation, channel quality estimation, retry limit adaptation, queue length estimation, etc. In [3], the cross-layer QoS-optimized EDCA adaptation algorithms take into account the unequal error protection characteristics of video streaming, the IEEE 802.11e EDCA parameters

${ }^{1}$ This work is supported by Qatar National Research Fund (QNRF) No. 08-374-2-144. and the lossy wireless nature. It makes use of two models, video distortion model and channel throughput estimation model to predict the video quality. The convex nature of the optimization problem remains an open research issue. The work of rate allocation becomes challenging since heterogeneity exists in both the rate utilities of video streams and in wireless link qualities. In a distributed manner, the task can be divided into sub-tasks, each can be performed independently by an individual node in the system, based on its perception to the system queue, and channel states. In [4], an optimization framework to distribute video rate allocation over wireless is proposed, taking into account this challenge. In [5] the authors investigate the packet loss behavior in the IEEE 802.11e wireless local area networks (WLANs) under various retry limit settings. Considering scalable video traffic delivery over the IEEE 802.11e WLANs, the presented study shows the importance of adaptiveness in retry limit settings for the Unequal Loss Protection (ULP) design. Based on the study, they present a simple yet effective retry limit based ULP which adaptively adjusts the retry limit setting of the IEEE 802.11e medium access control protocol to maintain a strong loss protection for critical video traffic transmission.

A new packet scheduler in cross layer environment for GSM/EDGE systems to improve QoS support of multiclast data services is proposed in [6]. The algorithm minimizes a prescribed cost functions given the current channel qualities and delay states of the packets in the queue. A cross-layer optimization for video streaming over wireless multimedia sensor networks is attempted in [7]. In 802.11s mesh networks, packets are differentiated and higher priorities are given to forward packets.

Weighted Fair Queuing [8] is efficient for wireless channels. It assigns weight for different flows and calculates the departure time based on the weights. Assigning weight to the individual flows helps in prioritizing the video packets and sending the packets in the flow which has more weight.

Forward Error Correction [9] is used to reduce the number of packets lost. This is done by adding redundant number of packets to the video sequences. The challenge is to add optimum number of packets suitable for both channel availability and queue length. An adaptive video packet scheduling algorithm used in WLAN is proposed in [10]. The data transmitted over the wireless channel should be reduced as much as we can considering the limitation of wireless bandwidth, but not the video-quality. If the network load becomes higher and higher, the access point must compare the multiple video streams and find which one should be transmitted first.

Unlike previous works, this paper addresses a distributed sequential mapping strategy based on EDCA access scheme using symantec-based video quality metric that measures statistically the number of effective reconstructed video layers. We aim to improve video quality that can be affected by channel contention. The basic approach to delivering the video stream over EDCA consists of mapping all the video packets to one AC. This approach has two obvious 
drawbacks when considering high channel contention scenario. The first, it causes queue overflow and increases the number of dropped packets at the interface queue. Second, all layers at different users are mapped to the same $\mathrm{AC}$, that is, they will use the same Arbitration Inter-Frame Spacing (AIFS), which increases the probability of collision. Hence mapping the layers to different $\mathrm{AC}$ queues decreases the interface queue drops and allows different priorities to the different video layers based on their dependencies.

This paper proposes a dynamic programming-based algorithm that takes into account different layer rates and channel conditions to estimate the optimal mapping strategy between the video layers and the 802.11e EDCA priority queues. We model the mapping between a multi-layer video delivery, such as the one employed in the ubiquitous H.264, and 802.11e. We motivate our work through absence of a similar model and/or lack of attention to specific layering properties. With theoretical analysis, we derive the Estimated Reconstructed Video Layers (ERVL) taking into account the wireless channel contention model and the video layer dependency. We define the canonical mapping strategies: a subset of the mapping strategies to enhance the performance of searching for optimal video layers mapping strategy, and show that given the dependency between the video layers, the optimal mapping strategy must belong to the canonical mapping subset. We also provide proof for the lemma and study the complexity of our proposed algorithm. The results of the extensive set of simulations that we performed show the efficiency of the distributed sequential mapping proposal to maximize the average number of useful layer perceived. The problem of distributed synchronized mapping has been addressed in [11]. There, it was assumed that 1) the video layers have equal rates, and 2) there is a perfect synchronization amongst users in calculating the optimal mapping strategy. However, in order to realize such a technique in practical settings, we assume that the access point would constantly signal all users at the same time to perform the mapping enumerations. While these assumptions may sound realistic, they reduce the chances of adopting such a technique in practice, and therefore, in this paper, we relax these assumptions by eliminating the equal rates, and perfect synchronization requirements, which also removes the requirement to have constant signaling from the AP to all users at the same time.

The remainder of this paper is organized as follows. We devote Section II, for depicting the overall system model including the description for EDCA channel and layered video mapping technique. We provide a deeper analysis of the main obtained results in Section III. Section IV summarizes the paper and outlines the future works.

\section{SySTEM MODEL}

This Section describes the analytical study of the optimal cross-layer wireless layered video delivery approach. Our solution is called Distributed Sequential Mapping (DSM). It is based on the IEEE 802.11e EDCA scheme which provides a differentiated access to the medium using different priorities for different types of traffic [2], briefed in the following subsection. We consider a layered video source encoded into a base layer and multiple enhancement layers, as shown in Figure 1. In our study, we consider that the sources of video layers have different bitrates. We aim to achieve the best video quality by maximizing the number of received video layer for each user, running a sequential algorithm, in which, one user at a time, after being signaled by AP, tries to search for the optimal mapping strategy using a snapshot of the system parameters, including the current mapping strategies of all other users, and the wireless channel condition. Although each user estimates the optimal mapping strategy locally, we show that after second round of running the sequential algorithm, the achieved solution is very close, and in some cases matches the global optimal solution assuming perfect synchronization.

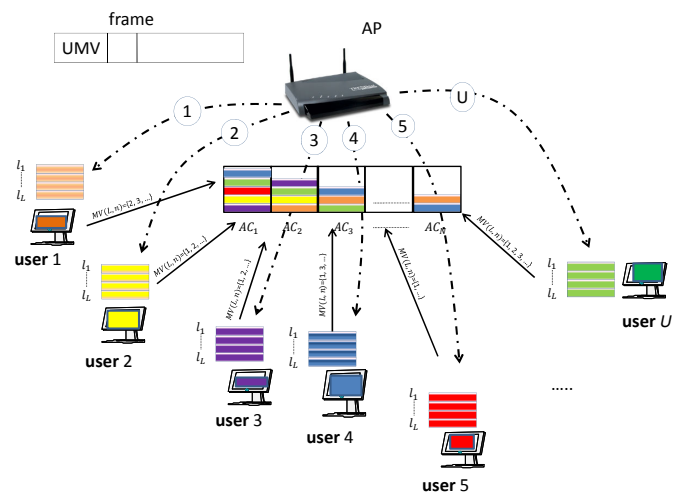

Figure 1. Distributed Sequential Mapping Model

\section{A. EDCA Analytical model}

Basically, an EDCA channel access function uses Arbitration Inter-frame Space $(A I F S[A C])$, Contention Window, with its minimum and maximum values, $C W_{\min }[A C]$ and $C W_{\text {max }}[A C]$ respectively, for the contention process to transmit a packet that belongs to an $A C$. These parameters can be used in order to differentiate the channel access among different priority traffic. The channel access priority goes from $A C_{4}, A C_{3}, A C_{2}$, up to the highest priority $A C_{1}$. As the priority of the $A C$ increases, the values of the MAC parameters become smaller. Therefore, the $A C$ with the shorter contention period has more priority to occupy the channel. In this subsection, we present a simple EDCA model under saturation condition as described in [12]. This model estimates the following: 1) the collision probability that includes both internal and external collisions, 2) the interface queue dropping probability that computes the packets drop due to queue overflow, and finally 3) a delay model that accounts for all events that contribute to the access delay. These parameters capture the influence of the $C W \min \left[A C_{i}\right]$ and $C W \max \left[A C_{i}\right], A I F S$, and Transmission Opportunity $(T X O P)$ mechanisms.

We assume that we have $U$ video users (or subscriber stations SS), and $n_{A C_{i}}$, denote by the number of ACs for all users contending for the channel, such that each layer from one video user is assigned to one $A C_{i}$. We consider the Markov chain introduced in $[13,12]$. We extend the probability formulas to support differential TXOP Limit parameter in the different computed performance metrics [14]. In the following, we denote by $\tau_{i}$ the probability that a node in the $A C_{i}$ transmits during a generic slot time and by $p_{i}$ the probability that $A C_{i}$ senses the medium busy. The $\tau_{i}$ takes into account both internal and external collision. We follow the basic EDCA backoff increase scheme [13]. From the point of view of one wireless node, the probability $\tau$ that a node transmits in a given slot is:

$$
\tau=1-\left(\prod_{i=1}^{4}\left(1-\tau_{i}\right)\right)
$$

The collision probability due to both internal and external collisions for an $A C_{i}$, is defined as follows:

$$
p_{\text {coll }, i}=1-(1-\tau)^{U-1} \prod_{h \prec i}\left(1-\tau_{h}\right)
$$

With both collision and transmission probabilities, we can compute the probability $p_{s u c c, i}$ that a slot contains a successful transmission of frame of $A C_{i}$. The probability $p_{\text {succ }, i}$ 
is given by:

$$
p_{\text {succ }, i}=U * \tau_{i}\left(1-p_{\text {coll }, i}\right)
$$

According to $[13,14]$ the throughput is:

$$
S_{i}=\frac{p_{\text {succ }, i}\left(K_{T X O P_{i}}+1\right) E\left[l_{d}\right]}{\left(1-p_{\text {busy }}\right) \theta+p_{\text {succ }} T_{S_{i}}+p_{\text {coll }} T_{C}}
$$

Where $E\left[l_{d}\right]$ is the average data packet length, $p_{\text {succ }}$ is the probability that a station transmits successfully, $p_{\text {coll }}$ is the probability that a collision occurs for station, $T_{S_{i}}$ is the transmission time, $T_{C}$ is the collision time, $K_{T X O P_{i}}$ is the number of packets transmitted during transmission opportunity period, and $\theta$ is the duration of the slot time. The access delay for each $A C_{i}$ is defined as:

$$
E\left[D_{i}\right]=\frac{E\left[P_{i}\right]}{S_{i}}
$$

The dropping probability $P_{i, d r o p}$ of each $A C_{i}$ is:

$$
\begin{gathered}
P_{i, \text { drop }}=1-\left(\left(1-P_{i, \text { drop }, \text { coll }}\right)\left(1-p_{\text {queue }_{\text {drop }, i}}\right)\right) \\
P_{i, \text { drop }, \text { coll }}=p_{i}^{L_{i, \text { retry }}+1}
\end{gathered}
$$

Where $P_{q_{d, i}}$ is the probability that a packet is dropped due to the queue overflow, and $P_{i, d r o p, c o l l}$ represents the probability of frame drops due to maximum retry limit [12]. Let $K$ be the maximum size of the queue, and $\lambda_{i}$ is the application rate of an $A C_{i}$. We assume an exponential arrivals and departures of packets in the queue. Therefore, $P_{q_{d, i}}$ can be calculated based on the $\mathrm{M} / \mathrm{G} / 1 / \mathrm{K}$ state transition diagram as has been used in [15].

\section{B. Distributed sequential video delivery model}

Considering video coding schemes such as H.264/AVC, the video content is partitioned into sequences of pictures, referred to as groups of pictures (GOPs), each beginning with an independently decodable intra-coded picture. Each GOP contains many pictures or frames, and it is divided into a sequence of packets for delivery over the network. In layered coding, the video content is partitioned into multiple layers of sub-streams, and hence each GOP can be thought of as individual streams of packets, one for each layer. Layered video concept as MRC, divides the video into a base layer and multiple enhancement layers. The base layer can be decoded to provide a basic quality of video, while the enhancement layers are used to refine the quality of the video. If the base-layer is corrupted, the enhancement layers become useless, even if they are perfectly received. Therefore, receiving the $K$ th layer is only helpful if the previous $K-1$ layers have been received.

We aim to address an efficient mechanism to transmit video layers for $U$ users over wireless network. Sequential mapping is considered in order to make the algorithm more practical and scalable to the variable number of layers, while requiring no synchronization between users. The proposed optimal distributed sequential algorithm selects, for each user, a specific mapping strategy while considering fixed mapping strategy for other users. This algorithm aims to minimize the complexity of a synchronized solution while achieving a good video delivery quality.

In the DSM algorithm, the AP updates periodically the users by sending a frame including the overall traffic load and the latest mapping strategy vectors considered by all users during the previous period as shown in Figure 1. On one hand, this frame informs the users about channel activity which is useful to compute the dropping probabilities based on EDCA model. On the other hand, each user selects a new mapping strategy that maximizes the
ERVL delivered to the destination node, considering a fixed mapping for other users as described in the Users Mapping Vectors $(U M V)$ transmitted in the AP frame.

We define an arbitrary mapping vector for mapping $L$ layers to $n$ ACs as follows:

$$
M V(L, n)=\left\{x_{1}, x_{2}, . ., x_{l}, . ., x_{L}\right\}
$$

Where generally $L \geq n$ and $1 \leq x_{l} \leq n$ represents the index of the AC that layer $l$ is mapped into. We define the ERVL metric based on the individual dropping probability of each layer as the expected number of reconstructed video layers, given that layer $l_{i}$ can be reconstructed if and only if all layers $l_{1}$ to $l_{i-1}$ are received successfully. Therefore, we can formulate ERVL statistically as follows:

$$
\operatorname{ERVL}(M V(L, n))=\sum_{r=1}^{L} r * \prod_{i=1}^{r}\left(1-P r_{l_{i}}\right) * \prod_{h=r}^{L} \operatorname{Pr}_{l_{h}}
$$

Where $\operatorname{Pr}_{l_{i}}$ is the dropping probability of layer $l_{i}$ and $L$ is the maximum number of coded video layers represented by $V(L)=\left\{l_{1}, l_{2}, . . l_{L}\right\}$. The $\operatorname{Pr}_{l_{i}}$ is computed according to which $A C$ the layer $l_{i}$ is assigned to. Thus, all video layers mapped to the same $A C$ have the same dropping probability, assuming dropping is statistically independent across all layers. To estimate the $E R V L$ value, we need two nested loops to calculate the summation and the product, which deems the complexity of calculating $E R V L$ as $O\left(L^{2}\right)$. The $\operatorname{Pr}_{l_{i}}$ 's are pre-computed for all $r=1, \ldots . L$ according to the $A C s$ packet dropping probabilities $\left(P_{j, \text { drop }}\right.$, see Eq. 6$)$. Each user $u$, extracts from the $U M V$, the total number of active $A C s$ and the number of users contending for medium access. Then, it computes the dropping probabilities of each $A C$ based on EDCA model as described in the previous subsection, considering the channel load, and the information about users and active $A C s$. Let $N$ be the maximum number of $A C s:\left\{A C_{1}, A C_{2}, . . A C_{N}\right\}$ (for basic EDCA, $N=4$ ). We aim at developing strategies to efficiently map $V(L)$ to different set of $A C s$ in order to increase the $E R V L$ metric for each video user.

The exhaustive mapping defines all possibilities of mapping vectors: $\Delta(M V)=\{M V(L, n): 1 \leq n \leq N\}$. Thus, each user calculates, for each $M V(L, n)$, the $E R V L$ received at the sink node considering that other users are using a fixed mapping, as described in the AP frame. Then, it selects the best mapping strategy vector $M V^{*}(L, n)$ regarding the maximum estimated value of $E R V L$. Each user has to perform locally an exhaustive search algorithm to define the best mapping vector among $\Delta(M V)$. The Complexity $C_{E x S}$ of performing such exhaustive search, when considering $N$ $A C s$ and $L$ layers, is:

$$
C_{E x S}=\sum_{i=1}^{N}\left({ }_{L-i}{ }^{L-1}\right)
$$

We deduce from Equation (10) that $C_{E x S}=O\left(L^{N-1}\right)$, which has exponential complexity.

Canonical mapping: We define a canonical mapping strategy $M V_{c}(L, n)=\left\{x_{1}, x_{2}, x_{3}, . . x_{L}\right\}$ as an arbitrary vector, that maps $L$ layers to $n$ ACs, such that:

Ordered mapping: the $x_{i}$ values are always nondecreasing, that is $x_{i} \leq x_{j}, \forall i \leq j$.

Rate based mapping: the aggregate bitrate assigned to any $\mathrm{AC} A C_{i}$ is greater than or equal to aggregate bitrate assigned to $\mathrm{AC} A C_{j}, \forall i \leq j$.

If we assume that $y_{A C_{i}}$ is the number of layers assigned to $A C_{i}$, this means that for canonical mapping strategies, the first $y_{A C_{1}}$ are mapped into $A C_{1}$, then the following $y_{A C_{2}}$ to $A C_{2}$, etc., where $1 \leq y_{A C_{1}} \leq N$, and $\sum_{i=1}^{n} y_{A C_{i}}=L$. In other words canonical mapping vectors can be represented using the notation: $M V_{c}^{\prime}(L, n)=\left\{y_{A C_{1}}, y_{A C_{2}}, y_{A C_{3}}, . . y_{A C_{n}}\right\}$. Unlike mapping vectors defined by equation (8), notice that 
the canonical mapping vector $M V_{c}^{\prime}(L, n)$, is of size $n$, the number of active ACs.

We consider variable video data rate for each video layer, and for each user contending for channel access. Therefore, arbitrary canonical rate vector for a particular mapping strategy can be represented using the $R V_{c}^{\prime}(L, n)=$ $\left\{R_{1}, R_{2}, \ldots, R_{n}\right\}$, where $R_{i}=\sum_{k=Y_{i-1}+1}^{Y_{i}} r_{k}$ and $Y_{i}=\sum_{k=1}^{i} y_{k}$. Recall that in our MAC model we consider $A C_{j}$ has higher priority than $A C_{j+1}$.

Lemma 1 The optimal mapping strategy that guarantees maximum ERVL always belongs to the canonical mapping set, provided that the rates of the individual video layers are non-increasing i.e. $\forall R V(L), r_{i} \geq r_{j}, \forall i \leq j$.

Proof: See the proof in [11]

We describe the DSM algorithm, where each user sends video layers considering initial static mapping vector. The AP sends to all users, one by one, a frame including the last UMV and the channel load. When the user receives the frame, it selects the Optimal mapping vector considering the information included in the AP frame. The DSM algorithm is performed sequentially by the wireless users. Each user considers the latest mapping vectors selected by the previous users having already run the algorithm. A detailed description of the DSM algorithm for $L$ layers and $N A C s$ in Table I.

DSM Complexity study: We consider the worst case, where all layers have the same rate to calculate the computational complexity for each user. Let $C_{E R V L}$ be the complexity of computing ERVL described in Equation (9), $C_{D S M}$ the complexity of DSM algorithm, and $C_{L, N}$ the complexity of selecting the best mapping vector based on the maximum $E R V L$ having $L$ video layers and $N$ ACs: $C_{D S M}=C_{E R V L} * C_{M A P}$. When considering $4 A C s$, $C_{M A P}=1+\left\lfloor\frac{L}{2}\right\rfloor+\left(1+2+. .+\left\lfloor\frac{L}{3}\right\rfloor\right)+\ldots+\left(1+2+. .\left\lfloor\frac{L}{N}\right\rfloor\right)$ $\left(\lfloor x\rfloor\right.$ is the floor of $x$ ), leading to $C_{M A P}=O\left(N L^{2}\right)$. Thus, $C_{D S M}=O\left(N L^{4}\right)$. To reduce the complexity and the time necessary for completing the selection of the best mapping vector, we define a new dynamic program for calculating $E R V L$ recursively according to the number of active $A C s$ considered in the mapping strategy. We denote by $E R V L_{r}$ the ERVL metric value calculated recursively.

$$
\begin{array}{r}
E R V L_{r}(M V(L, n))=E R V L_{r}\left(M V\left(L-y_{A C_{n}}, n-1\right)\right) \\
* P_{n, \text { drop }}^{y_{A C_{n}}}+\prod_{h=1}^{n-1}\left(\left(1-P_{h, \text { drop }}\right)^{y_{A C_{h}}}\right. \\
\left.*\left\{\sum_{i=1}^{y_{A C_{n}}}\left(i+Y_{A C_{n-1}}\right) *\left(1-P_{n, \text { drop }}\right)^{i} * P_{n, \text { drop }}^{y_{A C_{n}}-i}\right)\right\}
\end{array}
$$

where $Y_{A C_{n-1}}=\sum_{j=1}^{n-1} y_{A C_{j}}$. Regarding Equation (11), $C_{E R V L_{r}}=O\left(\sum_{j=1}^{n} y_{A C_{j}}\right)=O(L)$, regardless of the number of active $A C s$ considered in the mapping strategy. Thus, this shows that the new derived complexity is lower than the complexity computed in Equation (9), by reducing complexity from $O\left(N L^{4}\right)$ to $O\left(N L^{3}\right)$ using pre-computation of the historical values of $E R V L_{r}$.

\section{MODEL VALIDATION}

In this section, we report analysis methodologies and results of the extensive simulations that have been done using Matlab. We consider layered video composed of $L$ layers. The EDCA parameters of all $A C$ are presented in Table I. Poisson distributed traffic consisting of 1024-byte packets was generated for each $A C$ regarding the selected mapping strategy. The Poisson traffic model has been considered in [15], among
Table I

MAC PARAMETERS FOR THE EDCA ACS.

\begin{tabular}{c|c|c|c|c}
\hline Parameters/ACi & $\mathbf{0}$ & $\mathbf{1}$ & $\mathbf{2}$ & $\mathbf{3}$ \\
\hline$C W_{\min }$ & 7 & 15 & 31 & 31 \\
\hline$C W_{\max }$ & 15 & 31 & 1023 & 1023 \\
\hline AIFS $[\mathbf{0 , 1 , 2 , 3}](\mu s)$ & 2 & 2 & 3 & 7 \\
\hline Max-retry limit $[\mathbf{0 , 1 , 2 , 3}]$ & 7 & 7 & 7 & 4 \\
\hline
\end{tabular}

several other papers, to model the multimedia traffic. Table I: DSM algorithm:

$\left(E_{\text {min }}(U), x_{\text {min }}(U)\right)=\operatorname{DSM}(L, N, U)$

For $u=1$ to $U$

$A P$ sends UMV to user $u$

I*At user $u^{* /}$

Adjust initial rates for all ACs based on info in UMV

$/ *$ Calculate $M V(L, N)$ for user $u$

$\left(E_{\text {min }}(u), x_{\text {min }}^{\prime}(u)\right)=$ Call $\operatorname{Map}(L, N)$

Communicate $E_{\min }(u)$, and $x_{\min }^{\prime}(u)$ back to AP

$\left(E_{\min }(u), x_{\min }^{\prime}(u)\right)=\operatorname{Map}(L, 1)$

$x_{\min }^{\prime}(u)=\{L\}$

Calculate $E_{\min } u$

$\left(E_{\min }(u), x_{\min }^{\prime}(u)\right)=\operatorname{Map}(L, 2)$

$x^{\prime}(u)=\{L, 0\}$

While $R_{A C_{2}} \leq R_{A C_{1}}$

Calculate $\operatorname{ERVL}\left(x^{\prime}(u)\right)$

If $\operatorname{ERVL}\left(x^{\prime}(u)\right)<E_{\text {min }}$

$E_{\text {min }}(u)=E R V L\left(x^{\prime}(u)\right)$, and $x_{\text {min }}^{\prime}(u)=x$

add one layer to $A C_{2} \rightarrow x=\left\{x_{1}-1, x_{2}+1\right\}$

$\left(E_{\text {min }}(u), x_{\text {min }}^{\prime}(u)\right)=\operatorname{Map}(L, k)$

$x^{\prime}(u)=\{L, 0, \ldots, 0\}$

While $R_{A C_{2}} \leq R_{A C_{1}}$

While $R_{A C_{3}} \leq R_{A C_{2}}$

While $R_{A C_{k}} \leq R_{A C_{k-1}}$

Calculate $\operatorname{ERVL}\left(x^{\prime}(u)\right)$

If $E R V L_{r}\left(x^{\prime}(u)\right)<E_{\min }$

$E_{\min }(u)=E R V L_{r}\left(x^{\prime}(u)\right)$, and $x_{\min }^{\prime}(u)=x$

add one layer to $A C_{k} \rightarrow x=\left\{x_{1}, x_{2}, \ldots, x_{k-1}-1, x_{k}+1\right\}$

add one layer to $A C_{3} \rightarrow x=\left\{x_{1}, x_{2}-1, x_{3}+1, \ldots, x_{k}\right\}$

add one layer to $A C_{2} \rightarrow x=\left\{x_{1}-1, x_{2}+1, \ldots, x_{k}\right\}$

We aim to evaluate the performance of the proposed DSM algorithm, executing two rounds. In the first round, the DSM algorithm is performed regarding an arbitrary initial mapping for each video user. However, in the second round, the mapping selected in the first round is considered by DSM to obtain optimal mapping for all users. We compare the DSM results with the ideal Synchronized Optimal Canonical Ordered Mapping (SOCOM) scheme [11]. We classify the obtained set of mapping strategies, to synchronized (SOCOM) and the non-synchronized (DSM) techniques. We aim to study the different parameters setting that have to be selected in order to enable high video delivery quality. The video quality is measured by the expected number of received layers.For each described mapping scheme, we compute ERVL metric as defined in the previous Section. We focus on this metric as it incorporates the effects of packet dropping rate and the access delay.

In the first set of simulations, we set $L=14$ and the maximum number of users $U=26$. We increase the number of users and we investigate the ERVL for both DSM and SOCOM algorithms. Figure 2 shows that SOCOM scheme provides the best video delivery quality when the number of nodes is more than 12. The DSM algorithm performance approaches to the global optimal achieved using the ideal SOCOM during the second round when the number of 


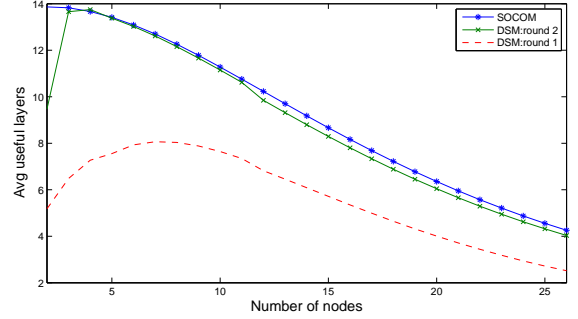

Figure 2. Performance comparison between SOCOM and DSM algorithms, for $\mathrm{U}=26, \mathrm{~L}=14$.

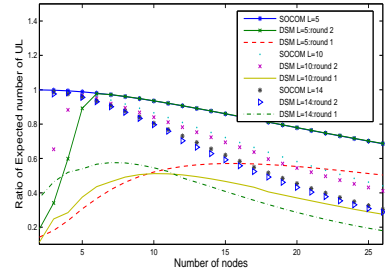

Figure 3. Ratio of the expected number number of useful layers $\mathrm{U}=26$, video delivery quality, $\mathrm{L}=14$.

nodes is less than 12. Furthermore, the obtained results show the evidence of video performance improvements of the sequential mechanism from the first round to the second one. This demonstrates the optimality and fast convergence of the DSM mechanism. Therefore, because of the DSM algorithm complexity is low, it becomes more practical comparing to the distributed synchronized solution (SOCOM).

We report in Figure 3 the impact of the number of video layers considered in the video coding. We present the normalized expected number of useful layers delivered to the destination node for $L=5,10$, and 14 . We observe that when the number of nodes is low, DSM (in second round) and SOCOM mechanisms provide a good performance regardless of the number of coding video layers. However, when the traffic load increases, the highest video delivery quality is achieved with a low number of layers. Indeed, for 26 nodes, about $70 \%$ of layers are delivered when $L=5$. However, only $40 \%$ of the layers are delivered when the video stream is coded to 14 layers $(L=14)$.

Now, we study the impact of video data rate on the DSM algorithm considering low and high application rates. Figure 4 shows that the best results are obtained with high application data rate. This ensures that DSM scheme is more adaptable to high network load.

Figure 5, shows the effect of setting different TXOP values on the DSM algorithm. We allow the user that has access to the medium to transmit more than one video packet without having to contend for access to the channel. We observe that, the TXOP has no significant effect when the traffic load is high. However, when we increase the TXOP value the performance of DSM decreases for low number of nodes. That is, considering large transmission opportunity duration for an $\mathrm{AC}$, increases the access delay for other Acs. Therefore, the packet dropping at the interface queue increases. Consequently, the EVRL decreases as it is affected by the packets drop rate.

Figure 6, shows the effect of varying the number of ACs on the video quality. The results show that the highest video delivery quality is obtained when increasing the number of ACs. However, considering more than 4 ACs per node becomes inefficient as ERVL changes become insignificant, while complexity increases $\left(C_{D S M}\right.$ is proportional to $\left.n_{A C}\right)$. This ensures the benefits of addressing dynamic mapping strategy mechanism.
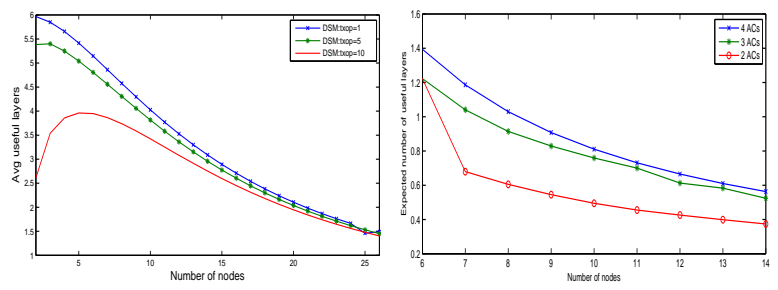

Figure 5. Impact of TXOP Figure 6. Impact of the numparameter on video delivery,for ber of access categories on video $\mathrm{U}=26, \mathrm{~L}=14$ delivery,for $\mathrm{L}=8$.

\section{CONClusion And Future WORK}

In this paper, we proposed a distributed sequential layered video mapping technique, over EDCA ACs. The proposed algorithm dynamically maps video layers to EDCA's appropriate ACs sequentially to reduce the signaling overhead from the AP to all participating users. The optimal mapping strategy was selected based on the maximum expected number of useful layers delivered for each sending user. We showed that canonical mapping strategies with variable layer rates ensure the best performance compared to other different mapping possibilities, especially for high application data rate. The obtained results showed that the described algorithm helps in meeting the performance improvement and also in decreasing the signaling overhead compared to the synchronized version.

\section{REFERENCES}

[1] S. McCanne and al, "Low-complexity videocoding for receiverdriven layered multicast," IEEE Journ. Sel. Areas in Comm., vol. 15, no. 6, pp. 983-1001, August 1997.

[2] S.Kerry and al, "Wireless LAN Medium Access Control (MAC) and Physical Layer (PHY) specifications Amendment 8: Medium Access Control (MAC) Quality of Service Enhancements," IEEE Std 802.11e-2005, p. 2005, 1997.

[3] A.Dua and al, "Channel, Deadline, and Distortion (CD2) Aware Scheduling for Video Streams over Wireless," IEEE Trans. Wireless Comm., vol. 9, no. 3, March 2010.

[4] X.Zhu and B.Girod, "Distributed Media-Aware Rate Allocation for Wireless Video Streaming," IEEE Transactions on Circuits and Systems for Video Technology, vol. 20, no. 11, pp. 1462-1474, November 2010.

[5] S. McCanne and al, "Low-complexity videocoding for receiverdriven layered multicast," IEEE Journ. Sel. Areas in Comm., vol. 15, no. 6, pp. 983-1001, August 1997.

[6] K. Johnsson and D. Cox, "An Adaptive Cross-Layer Schedule for Improved QoS Support of Multiclass Data Services on Wireless Systems," IEEE Journal of Sel. Areas on Commun., vol. 23, no. 2, pp. 334-343, February 2005.

[7] J.Zhang and J.Ding, "Cross Layer Optimization for Video Streaming over Wireless Multimedia Sensor Networks," ICCASM, 2010.

[8] B.Dekeris and al, "Analysis of QoS Assurance Using Weighted Fair Queueing (WFQ) Scheduling Discipline with Low Latency Queue (LLQ)," ITI, June 2006.

[9] N.Harun and al, "Impact of Weight Values in Hybrid and Adaptive FEC Mechanism over Wireless Network," International Conference on Network Applications, Protocols and Services, June 2010.

10] R.Liu and al, "Adaptive Scheduling Algorithm for Video Streams in Wireless Local Area Networks," Pacific-Asia Conference on Circuits, Communications and System, 2010.

11] L.Romdhani and A.Mohamed, "An analytic study of a distributed EDCA-based QoS mapping for layered video delivery in WLAN," Accepted in PEMWN http://arxiv.org/abs/1304.4472, 2012.

[12] Yang Xiao, "Performance Analysis of Priority Schemes for IEEE 802.11 and IEEE 802.11e Wireless LANs," IEEE Trans. Wireless Comm., vol. 4, no. 4, July 2005.

[13] G. Bianchi, "Performance analysis of the IEEE 802.11 distributed coordination function," IEEE Journal of Sel. Areas on Commun. vol. 18 , no. 3, pp. 535-547, March 2000.

14] L.Romdhani and C.Bonnet, "Analysis and Optimization of the 802.11e EDCA Transmission Opportunity(TXOP) Mechanism," IEEE Wimob, 2007.

[15] Q. Li and M.Schaar, "Providing adaptive QoS to layered video over wireless local area networks through real-time retry limit adaptation," IEEE Trans. Multimedia, vol. 6, no. 2, pp. 278-290, April 2004

[16] L.Romdhani and al., "QUMESH: Wireless mesh network deployment and configuration in harsh environment,," IEEE WCNC, April 2012. 